Supporting information for:

Percolated ionic aggregate morphologies and decoupled ion transport in precise sulfonated polymers synthesized by ring-opening metathesis polymerization

Benjamin A. Paren ${ }^{1 . \|}$, Bryce A. Thurston ${ }^{2, \|}$, William J. Neary ${ }^{3}$, Aaron Kendrick ${ }^{3}$, Justin G.

Kennemur ${ }^{3}$, Mark J. Stevens ${ }^{2}$, Amalie L. Frischknecht*2, Karen I. Winey*1

${ }^{1}$ University of Pennsylvania, Dept. Of Materials Science \& Engineering, ${ }^{2}$ Center for Integrated

Nanotechnologies, Sandia National Labs, ${ }^{3}$ Florida State University, Dept. Of Chemistry \& Biochemistry

BB. A. P. and B. A. T. contributed equally to this work

*corresponding authors K.I.W. (winey@seas.upenn.edu) and A.L.F. (alfrisc@sandia.gov)

Table of contents

1. Temperature Modulated Differential Scanning Calorimetry (TMDSC)

2. X-ray Scattering Fit Example

3. Electrical Impedance Spectroscopy (EIS) Spectra Example

4. Structure Factor Calculation from MD

5. MD Analysis of Finite Size Effects

6. MD Snapshots

7. Radial Distribution Functions (RDF)

8. Graph Theory

9. Shape Anisotropy and Graph Cutoff Distance

10. MSD of cations, backbone carbons, and sulfonate groups

11. Force Field Charge Scaling 


\section{Temperature Modulated Differential Scanning Calorimetry (TMDSC)}

The reversible heat flow on the final cooling of each polymer is shown in Figure S1. A $T_{g}$ is not present in the temperature range accessed for the p5PhSA-Li and Na polymers. There is a possible $\mathrm{T}_{\mathrm{g}}$ of $286^{\circ} \mathrm{C}$ in p5PhSA-Cs. These results are consistent with previously reported DSC measurements of these materials. ${ }^{1}$

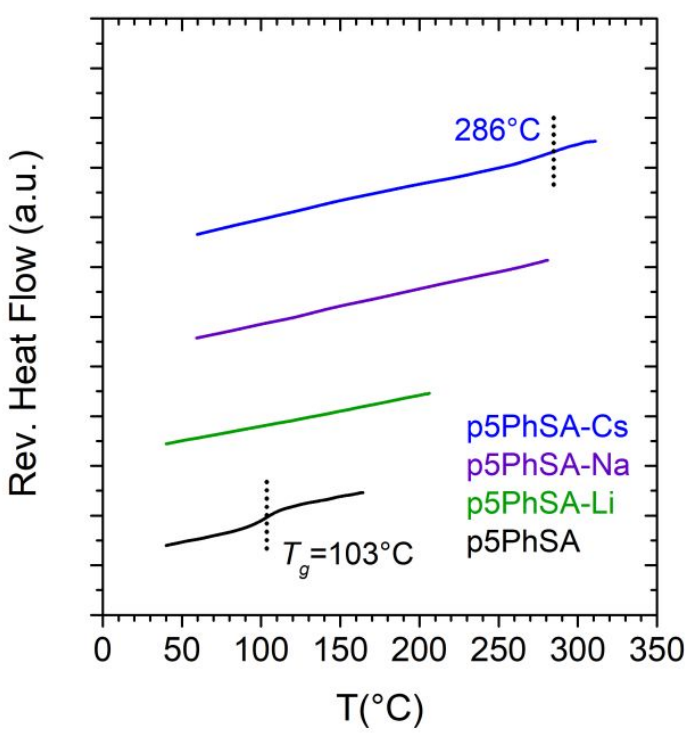

Figure S1. Reversible heat flow vs. temperature on cooling from temperature modulated DSC measurements of p5PhSA-X polymers. The acid form, p5PhSA (no neutralization), is included for reference. 


\section{X-ray Scattering Fit Example}

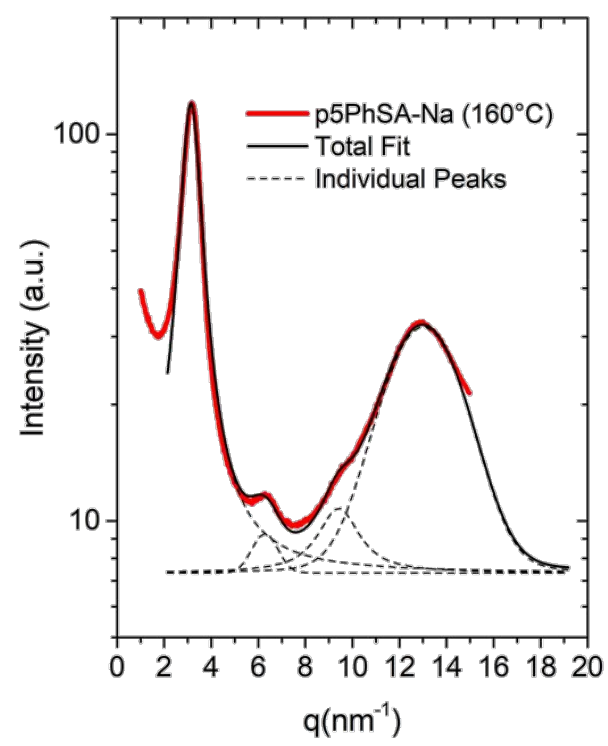

Figure S2. Representative example of fitting X-ray scattering data to pseudo-Voigt peak functions, used to extract peak positions. ${ }^{2}$ The total fit is the sum of the pseudo-Voigt fits of the 4 individual peaks.

Table S1. Primary peak positions and higher order peak ratios of the p5PhSAX polymers at $160^{\circ} \mathrm{C}$.

\begin{tabular}{|l|l|l|l|l|}
\hline Polymer & $\mathrm{d}^{*}(\mathrm{~nm})$ & $\mathrm{q}_{1}\left(\mathrm{~nm}^{-1}\right)$ & $\mathrm{q}_{2} / \mathrm{q}_{1}$ & $\mathrm{q}_{3} / \mathrm{q}_{1}$ \\
\hline p5PhSA-Li & 1.94 & 3.24 & 2.00 & 3.02 \\
\hline p5PhSA-Na & 1.99 & 3.16 & 2.02 & 3.05 \\
\hline p5PhSA-Cs & 2.09 & 2.99 & 2.01 & - \\
\hline
\end{tabular}




\section{Electrical Impedance Spectroscopy (EIS) Spectra Example}

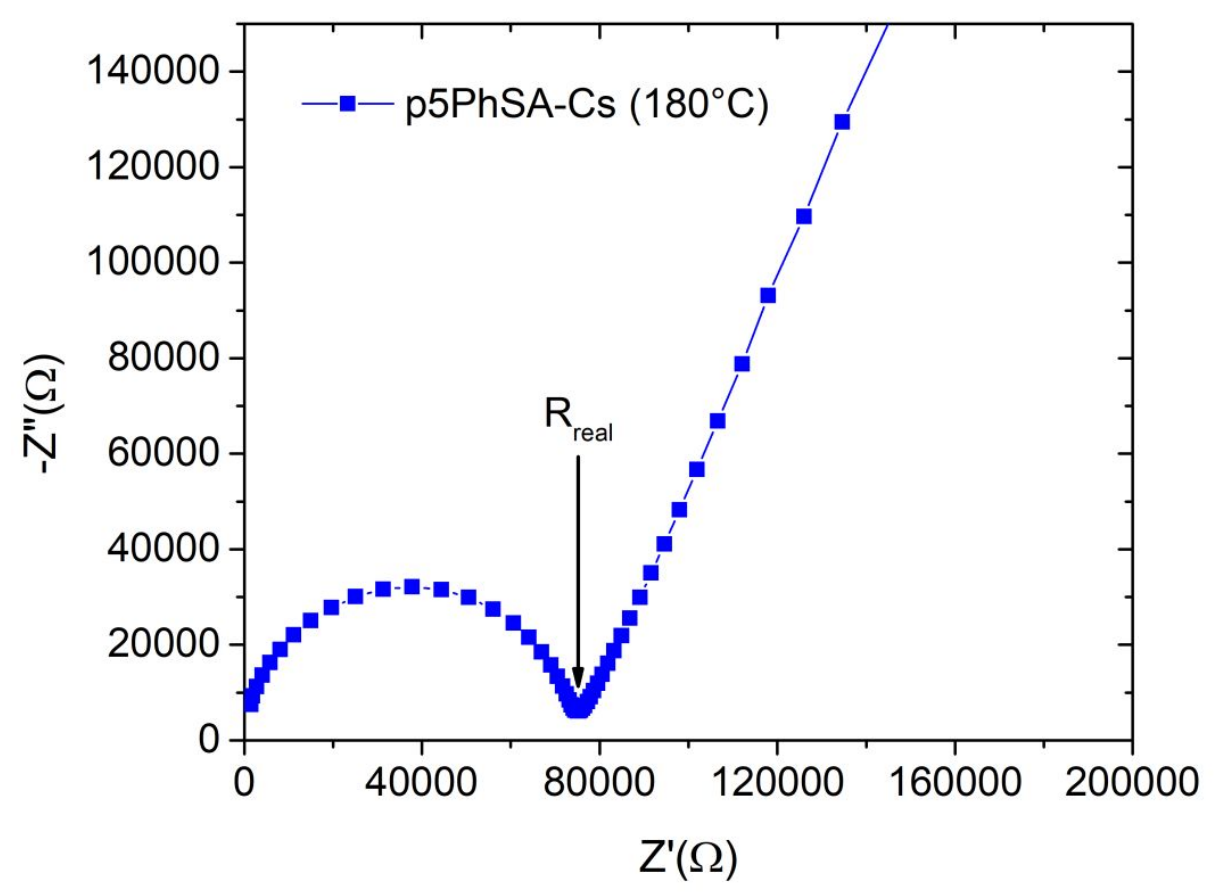

Figure S3. Representative Nyquist plot of p5PhSA-Cs at $180^{\circ} \mathrm{C}$. The value of Z' when $Z$ " is at a minimum is the resistance of the bulk polymer film 


\section{Structure Factor Calculation from MD}

We compute structure factors from our simulations as the Fourier transform of radial distribution functions $g_{i j}(r)$ :

$$
S(q)=\sum_{k}\left(c_{k} f_{k}(q)^{2}\right)+4 \pi \rho \int_{0}^{\infty}\left(w(r) \frac{\sin (q r)}{q r} r^{2} \sum_{i, j} c_{i} c_{j} f_{i}(q) f_{j}(q)\left(g_{i j}(r)-1\right)\right) \mathrm{d} r
$$

where $i, j$, and $k$ are sums over atom types, $c_{i}$ is the mole fraction of the $i$ th atom type, $f_{i}(q)$ is the form factor of the $i$ th atom type as a function of $q$, and $\rho$ is the atom number density. The modified Lorch weighting function $w(r)^{3,4}$ is

$$
w(r)=\frac{3}{(2 \pi r / L)^{3}}\left[\sin (2 \pi r / L)-\frac{2 \pi r}{L} \cos (2 \pi r / L)\right]
$$

and serves to smooth ripples resulting from the Fourier transform.

The functional dependence of the form factor on $q$ is approximated following Waasmaier and $\mathrm{Kirfel}^{5}$ as

$$
f_{i}(q)=a_{i}+\sum_{j=1}^{5} b_{i j} \exp \left(-d_{i j}(q / 4 \pi)^{2}\right)
$$

in which the functional dependence of a given atom type on $q$ is approximated as a sum of Gaussian functions using empirical parameters $a_{i}, b_{i j}$, and $d_{i j}$.

To assess the stability and accuracy of our simulations, we conduct several runs that vary the initial configurations, production run temperature, system size, and the ion charge. To study the effects of the initial configurations, we calculate $S(q)$ for a set of 4 systems each containing 216 polymers, smaller than the system used for the main results. Each distinct run has different residues sulfonated and different random initial orientations of each polymer. We follow this process for final run temperatures of $313 \mathrm{~K}\left(40{ }^{\circ} \mathrm{C}\right)$ and $433 \mathrm{~K}\left(160^{\circ} \mathrm{C}\right)$. The computed structure factors are shown in Figure S4, with error bars indicating the standard deviation over all runs. The relatively small size of the error bars indicates the low degree of variation as a function of the initial conditions. The low degree of variation between structure factors obtained at different temperatures illustrates the same temperature invariance as observed in experiment when $\mathrm{T}<<\mathrm{T}_{\mathrm{g}}$. 


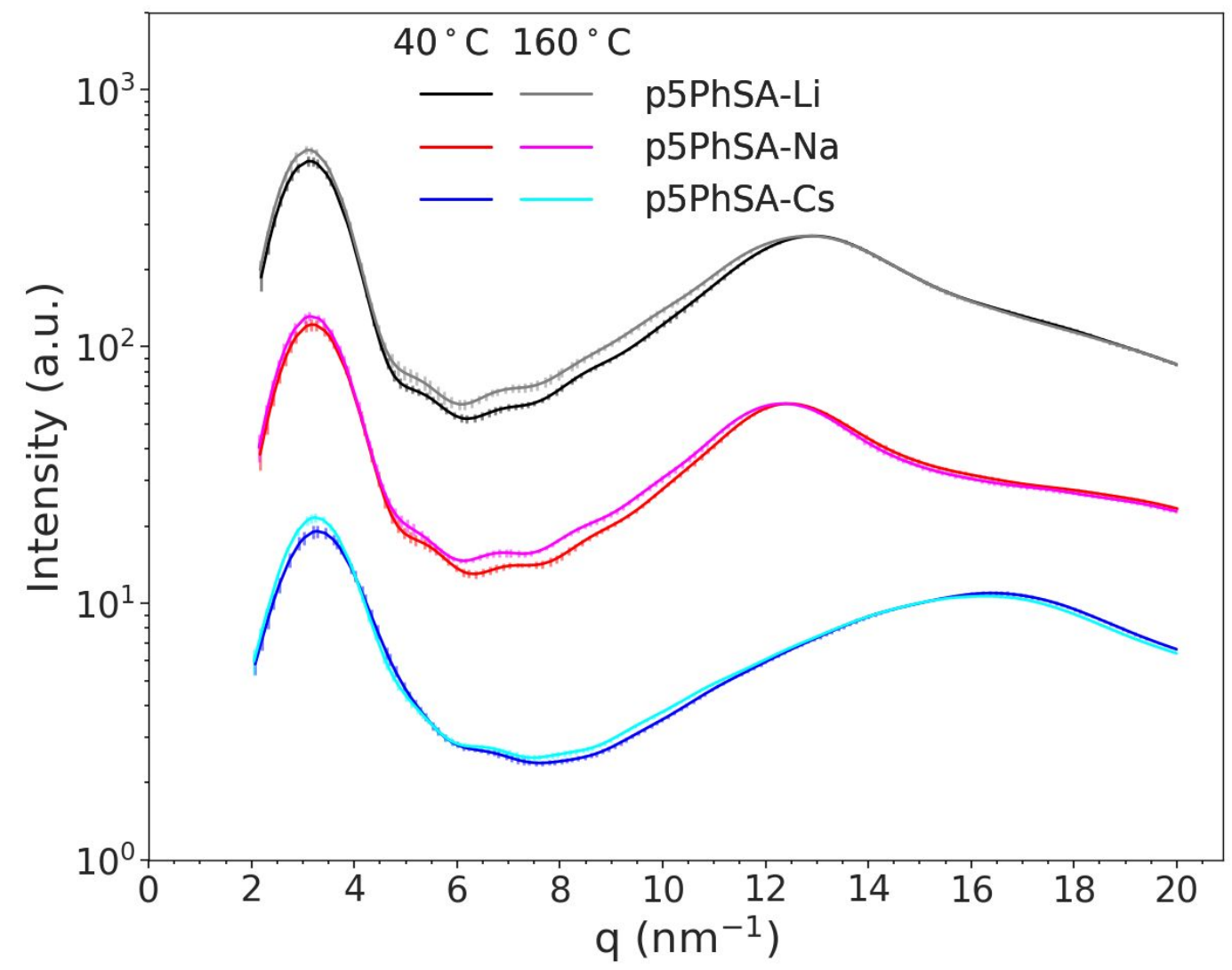

Figure S4. Computed average structure factors for $\mathrm{Li}, \mathrm{Na}$, and $\mathrm{Cs}$ ions at $40{ }^{\circ} \mathrm{C}$ and $160{ }^{\circ} \mathrm{C}$ from 4 distinct simulations, with 216 polymer chains each. Errors indicate standard deviations. As with experimental measurements, the structure factor is found to be insensitive to temperature in this range $\left(\mathrm{T}<<\mathrm{T}_{\mathrm{g}}\right)$. 


\section{MD Analysis of Finite Size Effects}

We also study the impact of finite size effects by simulating systems of $\mathrm{p} 5 \mathrm{PhSA}-\mathrm{X}$ with a variety of sizes. As in the main text, all simulations are conducted at $433 \mathrm{~K}\left(160^{\circ} \mathrm{C}\right)$ unless otherwise noted. We apply our simulation method for systems containing $\mathrm{n}^{3}$ polymers for $\mathrm{n}$ ranging from 4 to 12 for each ion species. Selected structure factors are shown in Figure S5. We find that as the system size is increased the simulated aggregate peak becomes increasingly sharp and becomes more intense relative to the amorphous halo. A weak higher-order peak appears for the largest simulation of the p5PhSA-Na polymer, though this small peak is likely smaller than the statistical error in $S(q)$. There is increasingly good agreement between simulation and experiment as the system size increases, indicating that finite size effects contribute to the discrepancies between computational results and experiment. In addition, force field imperfections and differences in density may also contribute to differences between experiment and simulations.
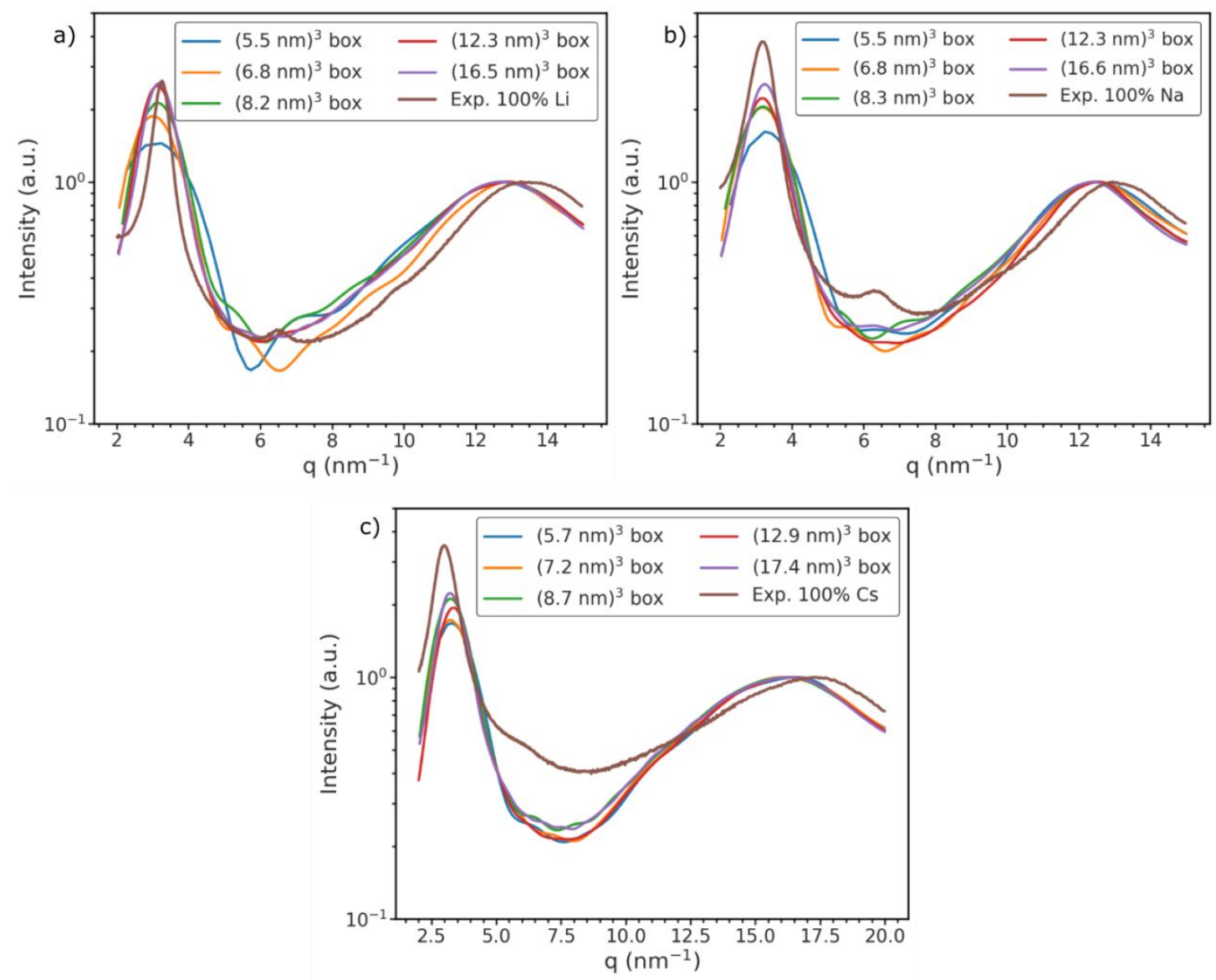

Figure S5. Structure factor as a function of box size for p5PhSA-X at $160{ }^{\circ} \mathrm{C}$ systems containing (a) Li, (b) $\mathrm{Na}$, and (c) Cs ions. Box sizes correspond to $\mathrm{N}=4,5,6,9$, and 12, where $\mathrm{N}^{3}$ is the total number of polymer chains. 


\section{MD Snapshots}

The atoms in the snapshots included in the body of the paper are colored by cluster. For the sake of clarity, we also include the snapshots with different colors for cations and oxygen atoms.

(a) p5PhSA-Li

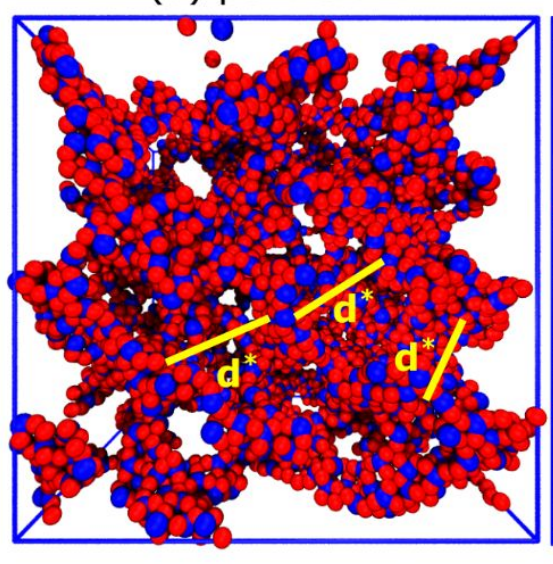

(b) p5PhSA-Na

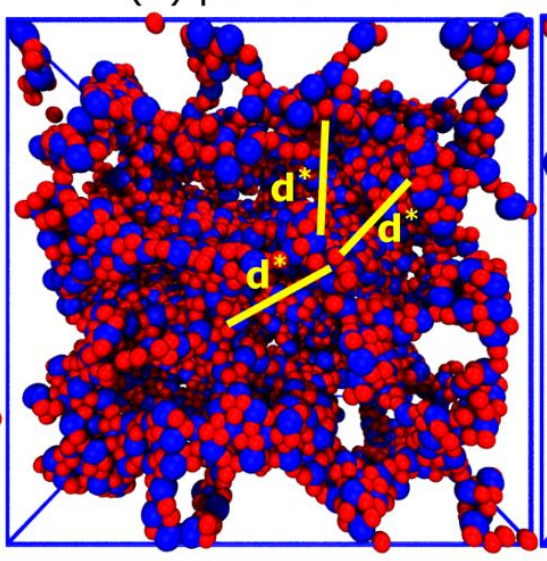

(c) p5PhSA-Cs

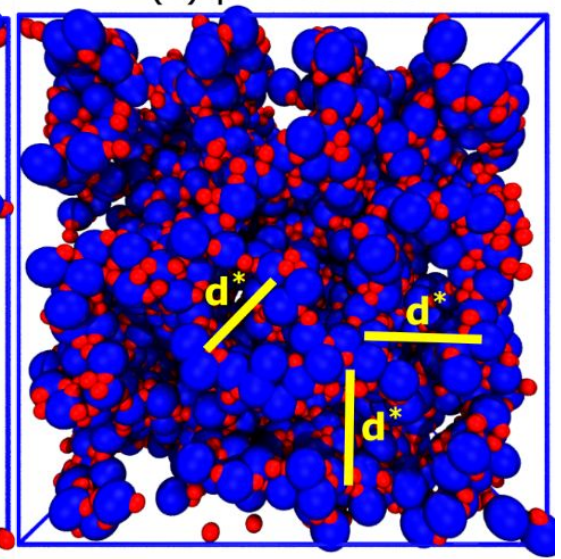

Figure S6. Snapshots of ionic aggregates observed in simulations. Identical to Figure 3, but all ions are colored blue and all oxygen atoms are colored red.

\section{Radial Distribution Functions (RDF)}

The partial radial distribution functions (RDF) for oxygen atoms with ions and with other oxygen atoms as well as for ions with ions are shown in Figure $\mathbf{S 7}$ for each ion species. In each system, the ion-oxygen RDF exhibits a sharp, clearly defined first peak. The first minimum in each of these systems, corresponding to nearest-neighbor pairs, is found to be $0.265 \mathrm{~nm}$ for $\mathrm{Li}, 0.34 \mathrm{~nm}$ for Na, and $0.425 \mathrm{~nm}$ for Cs. Similarly, the oxygen-oxygen RDF also exhibits a sharp first peak in each system. This corresponds to the distance between $\mathrm{O}$ atoms in the same sulfonate group. The first minimum in $g_{O O}(r)$ is found to be at $0.255 \mathrm{~nm}$ in each case. These minimum distances are used as the cutoff distances to define neighboring atoms for the structural analysis done in the body of the paper. Contrary to the Ion-O and O-O partial RDFs that consistently exhibit a clearly defined nearest-neighbor peak, the ion-ion RDFs have a significantly broader and less clearly defined first peak, especially in the case of the Li-neutralized polymer. As a result, using the ion-ion RDFs would not provide a clear way to define connectivity of the ionic aggregates. 


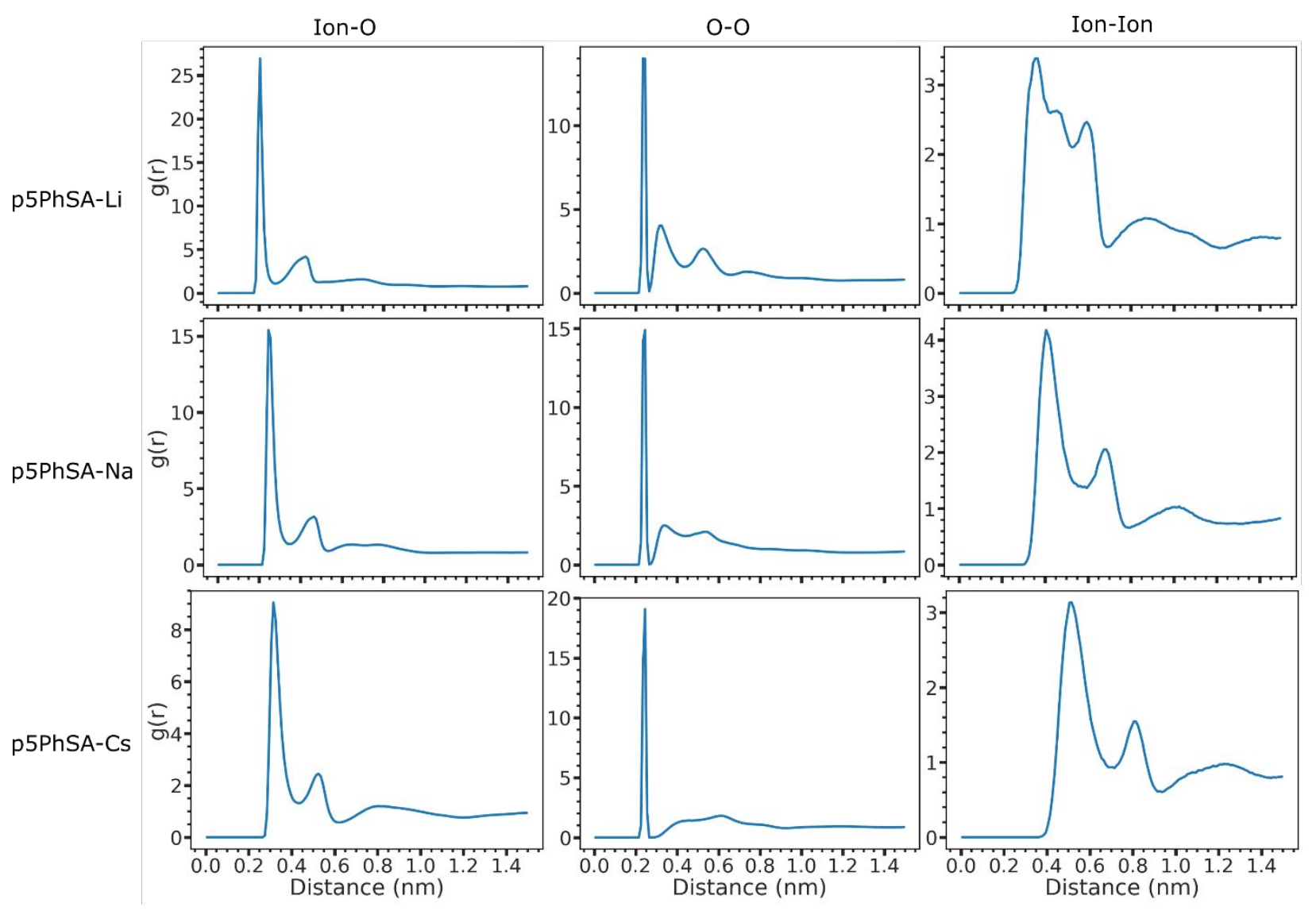

Figure S7. Radial distribution functions for each system for Ion-Oxygen, Oxygen-Oxygen, and Ion-Ion pairings of atoms. RDFs correspond to simulations of $\mathrm{p} 5 \mathrm{PhSA}-\mathrm{X}$ at $160{ }^{\circ} \mathrm{C}$ containing 216 polymer chains each.

\section{Graph Theory}

Graph theory involves the representation of objects or concepts that have a connected structure. ${ }^{6,7}$ Here, we are treating the structure of the ionic aggregates, whose connected structure is determined by atoms being unbonded neighbors as described in the main text. The atoms being modeled are represented in a graph as vertices. Connections between vertices are called edges. In this work the edges are unweighted, which means they all have the same unit length. The distance between two atoms is just the sum of the number of edges between the two atoms.

In the body of the paper, we represent the ionic aggregate formed by the oxygen atoms and ions as a graph. The conversion of the geometric configurations of the atoms to a graph-based representation is depicted in Figure S8. Every atom labeled in the geometric snapshot is labeled by the same letter in the graph, and every atom not labeled in the geometric configuration is not depicted in the graph. The circles in the graph representing the atoms correspond to vertices, and lines between them correspond to edges. 
A path on a graph corresponds to a series of vertices connected by edges in which no edge or vertex is repeated. For example, in the graph shown in Figure S7 a path from B to D might consist of $\{\mathrm{B}, \mathrm{E}, \mathrm{F}, \mathrm{H}$, $G, D\}$ or just $\{B, D\}$. The length of the path is the number of edges traversed between the two end vertices, so the path $\{B, E, F, H, G, D\}$ has a length of five and the path $\{B, D\}$ has a length of one. The distance between two vertices on a graph is the distance of the shortest path between the two. So, the distance between vertices $\mathrm{B}$ and $\mathrm{E}$ is one, between vertices $\mathrm{F}$ and $\mathrm{A}$ is three, and between $\mathrm{C}$ and $\mathrm{G}$ is two. Representing the ionic aggregate as a graph allows for the definition of a distance between two atoms that arises directly from the connectivity of the ionic aggregate. This distance only counts portions of the aggregate as being "nearby" if there exists a connected path between the two atoms.

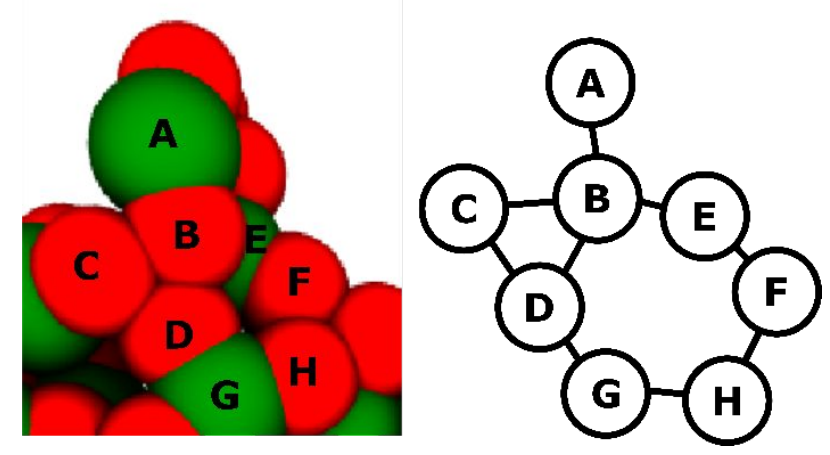

Figure S8. Illustration of the conversion of a small section of an ionic aggregate to a graph. Atoms labeled in the snapshot correspond to vertices labeled by the same letter in the graph. Unlabeled atoms in the snapshot are not shown in the graph. Circles in the graph correspond to vertices, and lines connecting vertices correspond to edges. Green spheres are Li ions and red spheres are $\mathrm{O}$ atoms. 


\section{Shape Anisotropy and Graph Cutoff Distance}

A graph-based cutoff distance of $\delta_{c}=10$ is used in the main text in the characterization of the local aggregate morphologies. Figure $\mathbf{S 9}$ plots the individual values $\lambda_{1}, \lambda_{2}$, and $\lambda_{3}$ for all the subclusters used in the calculation of $\kappa^{2}$ with $\delta_{c}=10$ (Figure 5e in the main text). The set of points corresponding to the subclusters in each system forms distinct clusters.
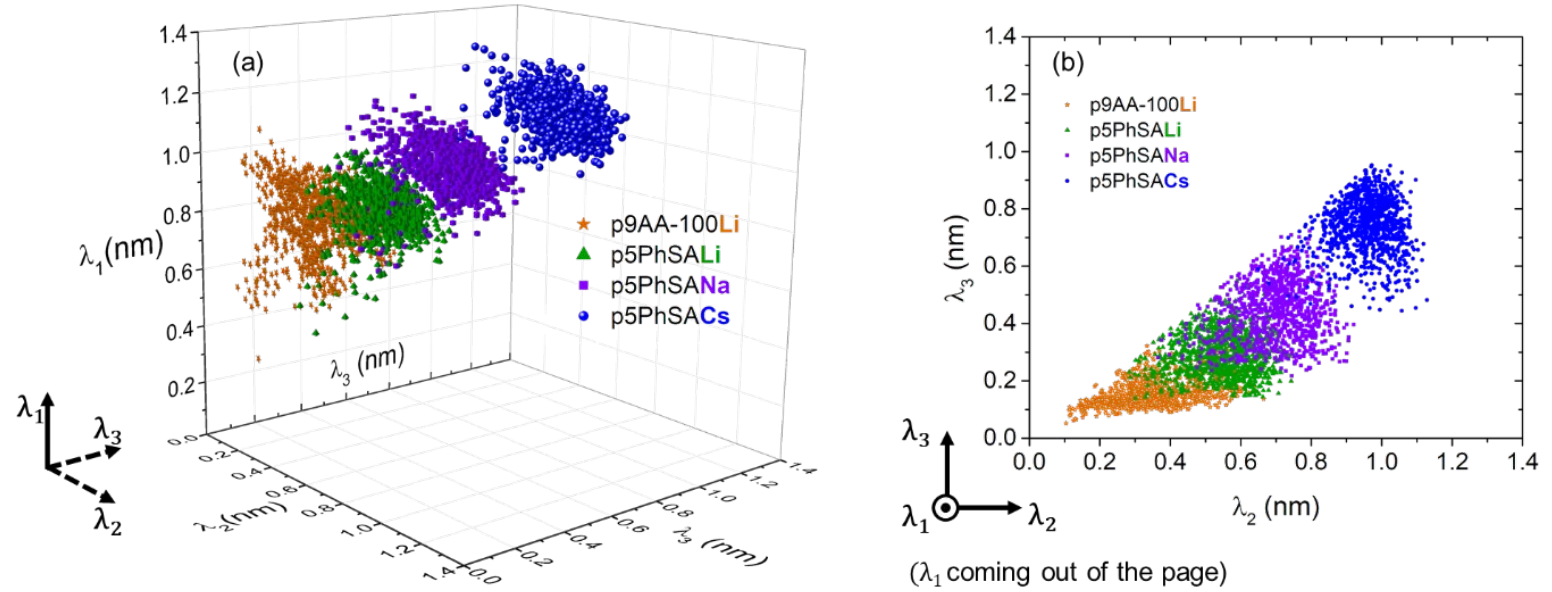

Figure S9. $\lambda_{1}, \lambda_{2}$, and $\lambda_{3}$ values for the p5PhSA-X and p9AA-100\% Li polymers. These values are used to calculate $\kappa_{i}^{2}$ (Equation 3 in the main text). Figures (a) and (b) contain the same data shown in two orientations for clarity. p5PhSA-X data correspond to systems at $160{ }^{\circ} \mathrm{C}$ containing 216 polymer chains.

We assess the consistency of the graph-based approach for extracting subclusters from the simulations by varying the graph cutoff distance. Figure $\mathbf{S 1 0}$ shows data for $\delta_{c}=7$ and $\delta_{c}=25$ that is consistent with $\delta_{c}=10$ in the main text. Histograms for the $\mathrm{p} 5 \mathrm{PhSA}-\mathrm{X}$ polymers exhibit similar shapes, with the median value of $\kappa^{2}$ decreasing as a function of ion size, while the p9AA-100\%Li system exhibits significantly larger values of $\kappa^{2}$ and a differently shaped distribution of values. As the $\delta_{c}$ value decreases from the value of $\delta_{c}=10$ chosen in the main text, the subclusters become less distinct and so less clearly anisotropic. On the other hand, increasing $\delta_{c}$ leads to large portions of the aggregate being included, so that the subclusters become increasingly isotropic. It is important to note that in computing the moments of the gyration tensor, we are taking linear projections of the geometry of the subclusters. The subclusters, however, exhibit some amount of curvature. This will tend to yield lower $\kappa^{2}$ values relative to those that would result in the case of zero curvature. 
(a)

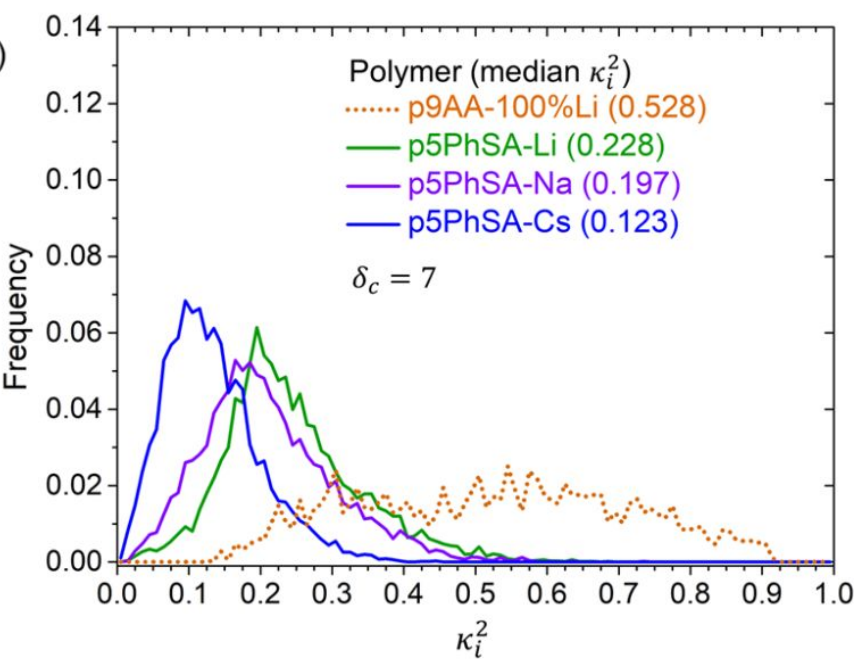

(c)

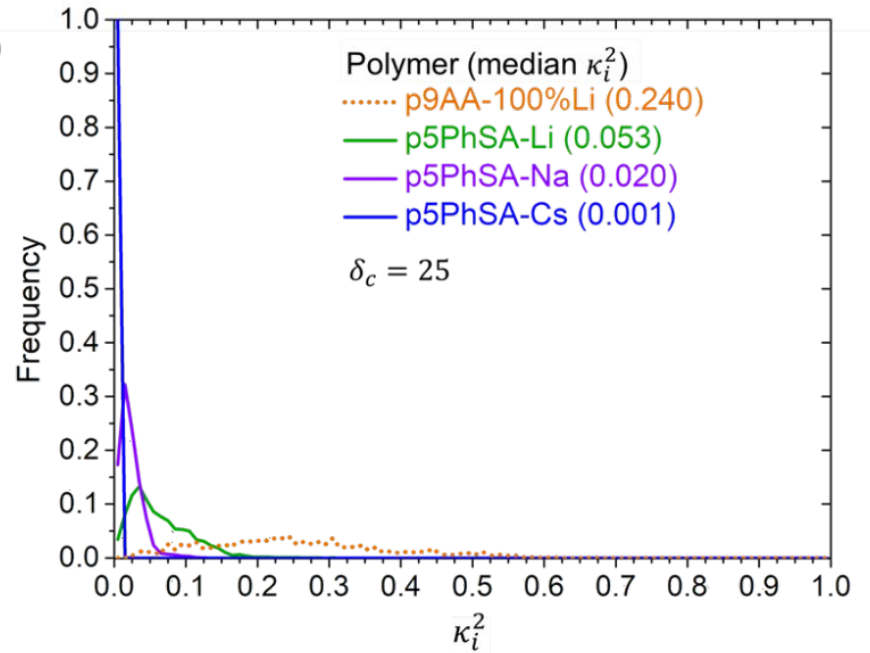

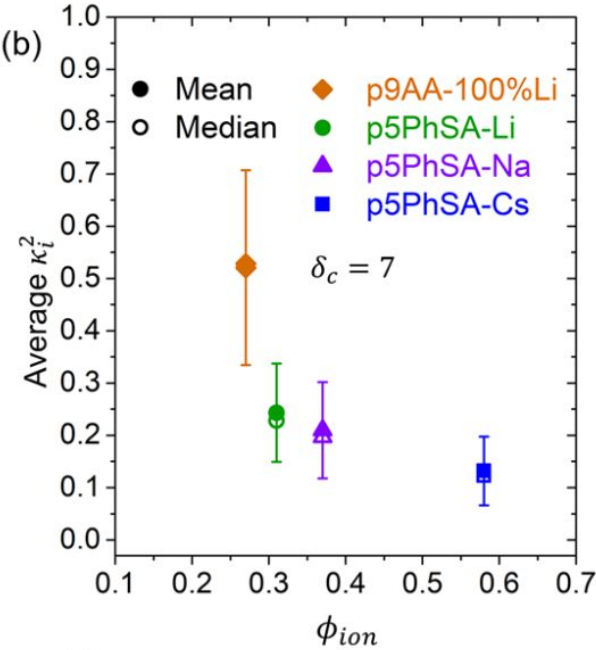

(d)

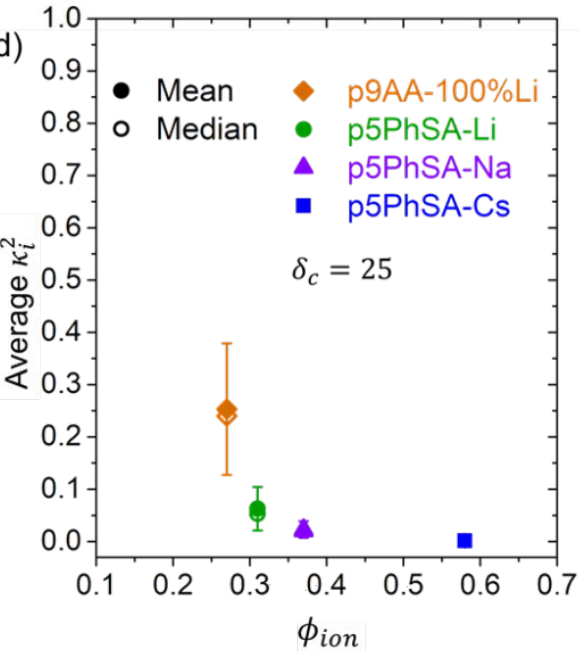

Figure S10. Distribution of $\kappa_{i}^{2}$ values for each ion species in p5PhSA-X and p9AA100\%Li for (a)-(b) $\delta_{c}$ $=7$ and (c)-(d) $\delta_{c}=25$. (a) and (c) show the distribution of $\kappa_{i}^{2}$ values, while (b) and (d) show the mean and median of $\kappa_{i}^{2}$ values vs. volume fraction of the ionic groups $\left(\phi_{i o n}\right)$, determined using the van der Waals (VDW) volumes of the atoms. Error bars correspond to the standard deviation of the mean. $\kappa_{i}^{2}$ distributions are from simulations with 216 polymers chains at $160^{\circ} \mathrm{C}$. The same trends are observed here as with $\delta_{c}=10$ in the main text (Figure 6). 


\section{MSD of cations, backbone carbons, and sulfonate groups}
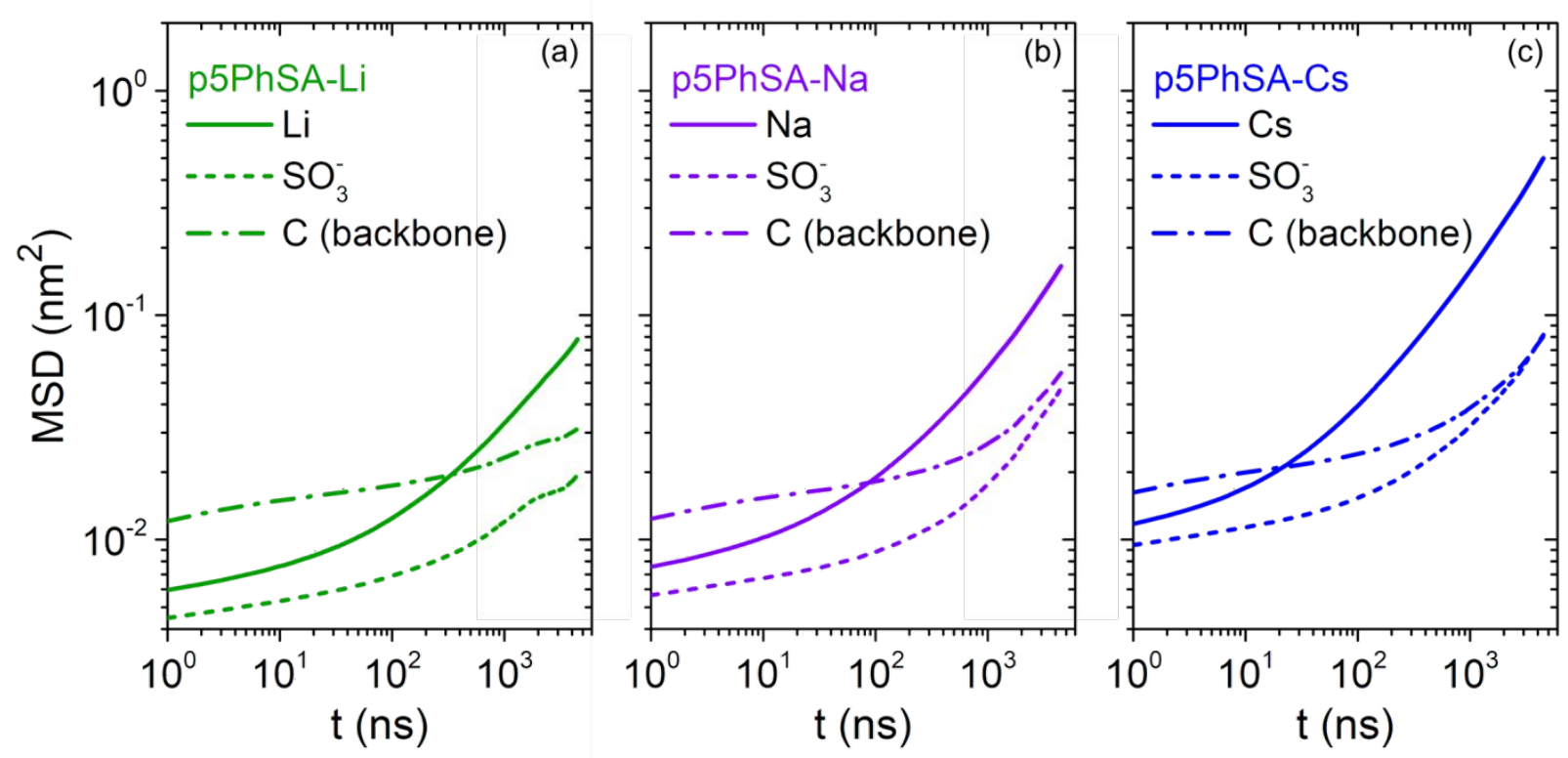

Figure S11. MSDs of cations, sulfonate groups, and polymer backbone carbons for each system. (a) p5PhSA-Li, (b) p5PhSA-Na, (c) p5PhSA-Cs. This data is from the same simulations analyzed in Figure 9 in the main text.

\section{Force Field Charge Scaling}

Several methods exist for explicitly accounting for polarization in classical molecular simulations, including charge fluctuation, induced point dipoles, and Drude Oscillators; ${ }^{8-10}$ however, each comes with a significant penalty to simulation speed. One approach to account for polarization effects to first order and to maintain higher performance is the Molecular Dynamics in Electronic Continuum (MDEC) method. ${ }^{11,12}$ This approach models the mean effects of polarization by rescaling the partial charges in the system by a constant value, which is functionally equivalent to dielectric screening by a constant factor. Charge scaling has often been shown to improve agreement between simulation and experiment, and is a common practice in MD simulations of a variety of systems, including ionic liquids. ${ }^{13-19}$ Leontyev and Stuchebrukhov recommend a charge scaling value of about 0.7 for ionic groups, ${ }^{12}$ though scaling by 0.75 and 0.8 are both quite common. ${ }^{13-19}$ To study the impact of taking this approach, we scale the charges of sulfonate groups and ions by a constant value of 0.75 . All other partial charges remain at standard OPLS-AA values.

From a structural standpoint, we find that this approach significantly degrades agreement with experiment. Figure $\mathbf{S 1 2}$ shows the computed structure factor for unscaled charges and scaled charges for a system of $12^{3}$ polymers. We find that scaling the charges significantly weakens the strength of the aggregate peak and shifts it to higher values of $q$ that are further away from the experimentally measured peak 
position. These differences are likely due to the weaker interactions between charges, producing ionic aggregates that are less tightly bound. In an attempt to improve agreement in structure between scaled charge simulations and experiment, we modified the annealing procedure. This was done by adopting the same annealing framework as described in the main text, but altering the temperature, pressure, and density parameters to produce optimal agreement in $S(q)$ between the Li neutralized system containing $6^{3}$ polymers and experiment. Optimization was conducted using the particle swarm optimization (PSO) procedure ${ }^{20-23}$ with global best topology and constant parameters $\omega=0.42, \phi_{1}=1.55$, and $\phi_{2}=1.55 .{ }^{23,24}$ See reviews for details on the method. ${ }^{22,23}$ Despite the fact that the optimization is conducted only for the p5PSA-Li polymer in a small system, Figure S12 illustrates that the PSO slightly improves agreement between the scaled system and experimental results. Nonetheless, the unscaled system produces better agreement with experiment, both in the aggregate peak position and the aggregate peak height relative to the amorphous halo. We thus conclude that scaling charges in the simulations leads to poorer agreement of polymer aggregate structure between simulations and experiment. 

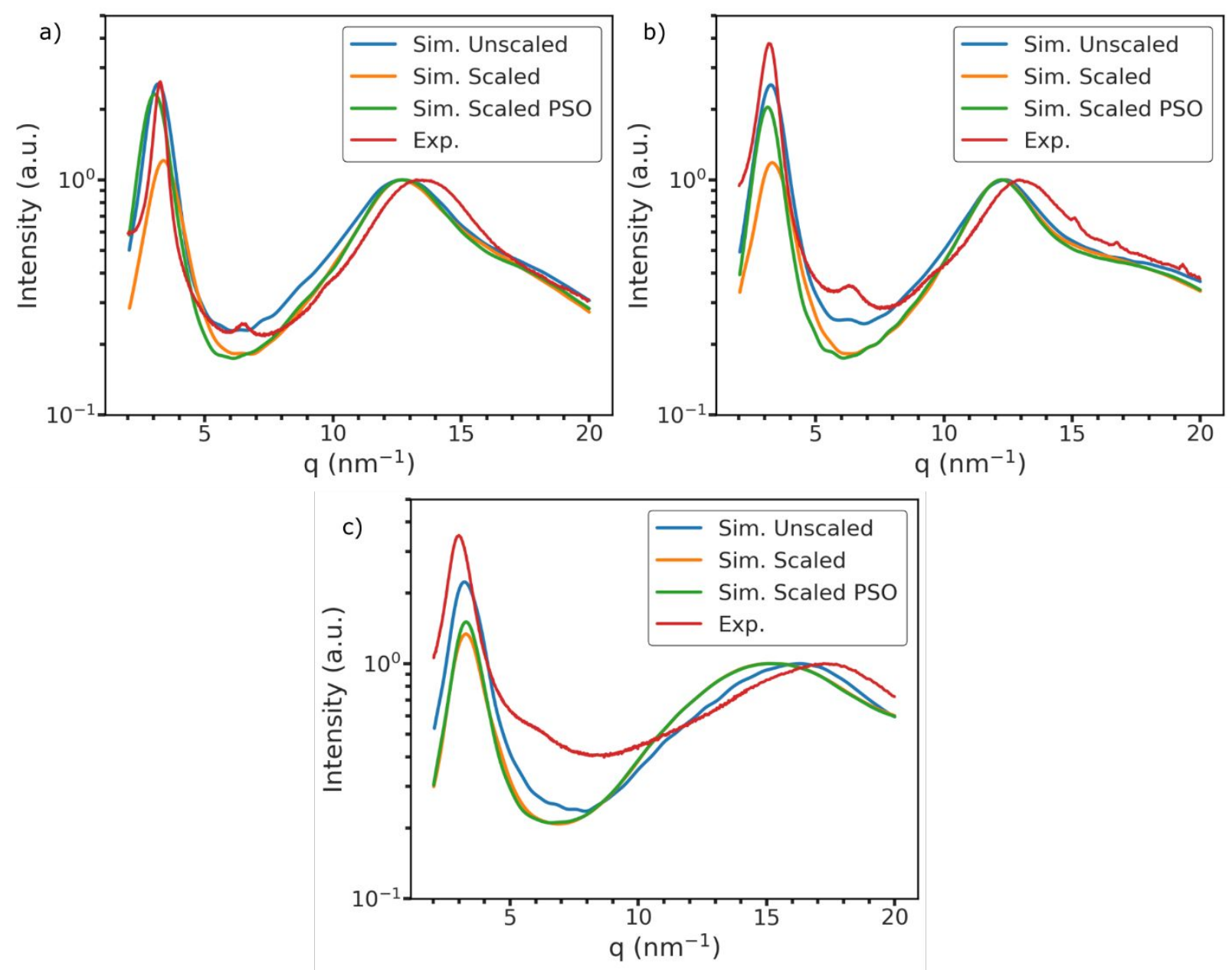

Figure S12. Simulated and measured structure factors for unscaled and scaled charges, for both standard annealing and PSO optimized annealing of p5PhSA-X for (a) Li, (b) Na, and (c) Cs ions. Each system contains 1728 polymer chains. While the PSO annealing procedure improves agreement with experiment, the systems with unscaled charges (blue) produce the best agreement between simulation and experiment (red).

The dynamics are also significantly altered by charge scaling. In scaled simulations, the ions are not as tightly bound to the sulfonate groups and thus diffuse through the system with greater ease. As a result, we are able to reach the diffusive regime for $\mathrm{Na}$ and $\mathrm{Cs}$ ions, and get significantly closer for Li ions (Figure S13). Specifically, the power law exponent relating MSD to $t$ is found to be $0.77,0.91$, and 0.96 for $\mathrm{Li}, \mathrm{Na}$, and $\mathrm{Cs}$ respectively. Given that $\mathrm{Na}$ and $\mathrm{Cs}$ both come quite close to reaching the linear regime, we extract a diffusion constant of $5.48 \mathrm{e}-9 \mathrm{~cm}^{2} / \mathrm{s}$ for $\mathrm{Na}$ ions and $4.69 \mathrm{e}-8 \mathrm{~cm}^{2} / \mathrm{s}$ for Cs ions. When we use the Nernst-Einstein equation to estimate conductivity from these diffusion constants, we obtain conductivities of $6.8 \mathrm{e}-5 \mathrm{~S} / \mathrm{cm}$ for $\mathrm{Na}$ and $4.9 \mathrm{e}-4 \mathrm{~S} / \mathrm{cm}$ for Cs. These are about three orders of magnitude higher than those measured in experiment. We are unable to extract conductivities from unscaled systems 
with similar methodologies. Thus, we conclude that scaling charges has an adverse impact on system dynamics. So, though it may yield improved simulation results in some systems, scaling the charges in these polymers leads to poorer performance of the simulations with respect to structure and dynamics. This may be because the ions form densely packed ionic aggregates in these polymers, and thus the electrostatics are inherently screened in the aggregates anyway, reducing the physical motivation for using scaled charges. We note that Zeron et al. argue that scaled charges are not appropriate for solids or molten salts, which the ionic aggregates resemble. ${ }^{25}$

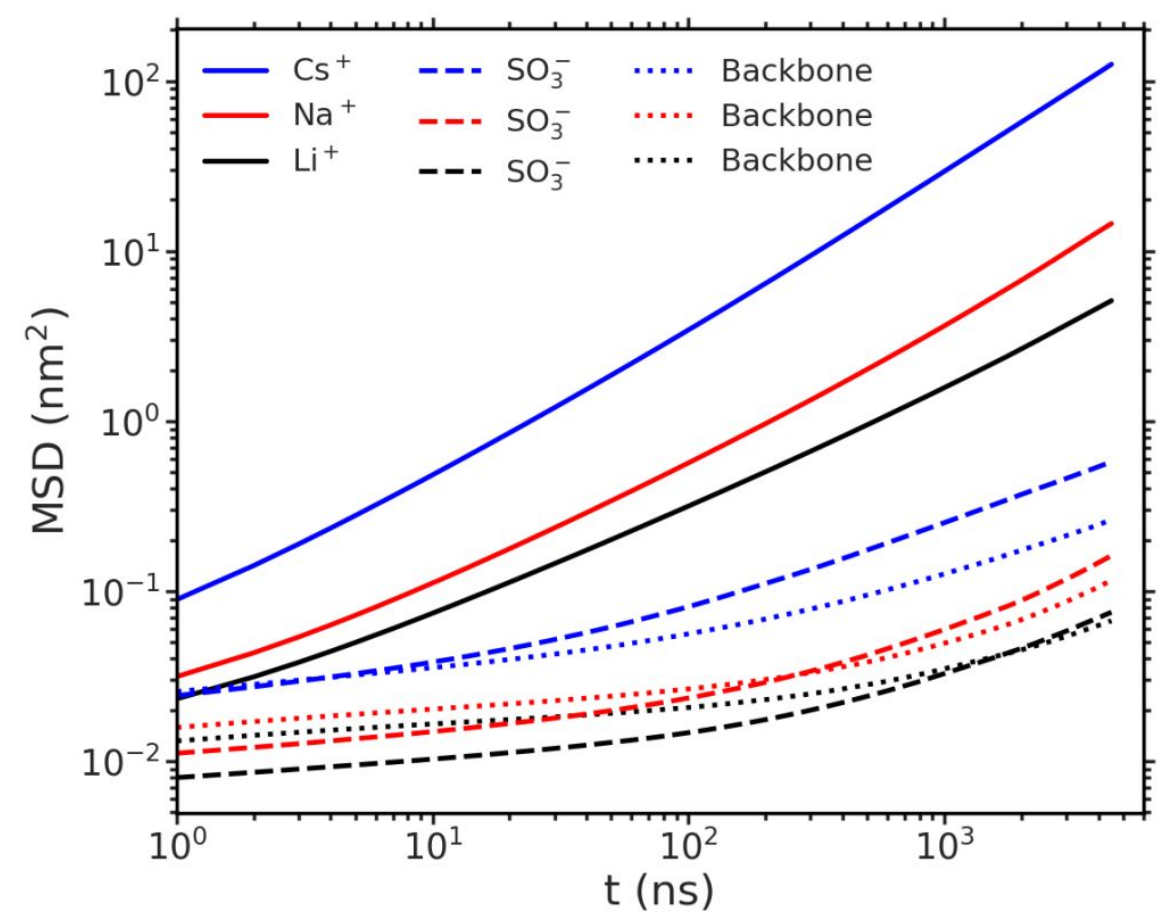

Figure S13. Mean squared deviations for cations, anions, and polymer backbone atoms for each p5PhSA$\mathrm{X}$ system with scaled charges at $160^{\circ} \mathrm{C}$. Na and $\mathrm{Cs}$ effectively reach the diffusive regime with exponents of 0.91 and 0.96 , and slopes corresponding to diffusion constants of $5.48 \mathrm{e}-9 \mathrm{~cm}^{2} / \mathrm{s}$ and $4.69 \mathrm{e}-8 \mathrm{~cm} / \mathrm{s}$, respectively. Each simulation contains 216 polymer chains. 


\section{References:}

(1) Kendrick, A.; Neary, W. J.; Delgado, J. D.; Bohlmann, M.; Kennemur, J. G. Precision Polyelectrolytes with Phenylsulfonic Acid Branches at Every Five Carbons. Macromol. Rapid Commun. 2018, 39, 1800145.

(2) Sánchez-Bajo, F.; Cumbrera, F. L. The Use of the Pseudo-Voigt Function in the Variance Method of X-Ray Line-Broadening Analysis. J. Appl. Crystallogr. 1997, 30 (4), 427-430.

(3) Lorch, E. Neutron Diffraction by Germania, Silica and Radiation-Damaged Silica Glasses. J. Phys. C Solid State Phys. 1969, 2 (2), 229-237.

(4) Soper, A. K.; Barney, E. R. On the Use of Modification Functions When Fourier Transforming Total Scattering Data. J. Appl. Crystallogr. 2012, 45 (6), 1314-1317.

(5) Waasmaier, D.; Kirfel, A. New Analytical Scattering-factor Functions for Free Atoms and Ions. Acta Crystallogr. Sect. A 1995, 51 (3), 416-431.

(6) Handbook of Graph Theory, 2nd ed.; Gross, J. L., Yellen, J., Zhang, P., Eds.; Discrete Mathematics and its Applications; CRC Press, 2014.

(7) Diestel, R. Graph Theory, 5th ed.; Graduate Texts in Mathematics; Springer-Verlag Berlin Heidelberg, 2017; Vol. 173.

(8) Halgren, T. A.; Damm, W. Polarizable Force Fields. Curr. Opin. Struct. Biol. 2001, 11 (2), 236-242.

(9) Lemkul, J. A.; Huang, J.; Roux, B.; MacKerell, A. D. An Empirical Polarizable Force Field Based on the Classical Drude Oscillator Model: Development History and Recent Applications. Chem. Rev. 2016, 116 (9), 4983-5013.

(10) Bedrov, D.; Piquemal, J.-P.; Borodin, O.; MacKerell, A. D.; Roux, B.; Schröder, C. Molecular Dynamics Simulations of Ionic Liquids and Electrolytes Using Polarizable Force Fields. Chem. Rev. 2019, 119 (13), 7940-7995.

(11) Leontyev, I. V.; Vener, M. V.; Rostov, I. V.; Basilevsky, M. V.; Newton, M. D. Continuum Level Treatment of Electronic Polarization in the Framework of Molecular Simulations of Solvation Effects. J. Chem. Phys. 2003, 119 (15), 8024-8037.

(12) Leontyev, I. V.; Stuchebrukhov, A. A. Electronic Continuum Model for Molecular Dynamics Simulations of Biological Molecules. J. Chem. Theory Comput. 2010, 6 (5), 1498-1508.

(13) Doherty, B.; Zhong, X.; Gathiaka, S.; Li, B.; Acevedo, O. Revisiting OPLS Force Field Parameters for Ionic Liquid Simulations. J. Chem. Theory Comput. 2017, 13 (12), 61316145.

(14) Mogurampelly, S.; Ganesan, V. Structure and Mechanisms Underlying Ion Transport in Ternary Polymer Electrolytes Containing Ionic Liquids. J. Chem. Phys. 2017, 146 (7), 074902.

(15) Chaudhari, M. I.; Nair, J. R.; Pratt, L. R.; Soto, F. A.; Balbuena, P. B.; Rempe, S. B. Scaling Atomic Partial Charges of Carbonate Solvents for Lithium Ion Solvation and Diffusion. J. Chem. Theory Comput. 2016, 12 (12), 5709-5718.

(16) Molinari, N.; Mailoa, J. P.; Kozinsky, B. General Trend of a Negative Li Effective Charge in Ionic Liquid Electrolytes. J. Phys. Chem. Lett. 2019, 10 (10), 2313-2319.

(17) Mason, P. E.; Jungwirth, P.; Duboué-Dijon, E. Quantifying the Strength of a Salt Bridge by Neutron Scattering and Molecular Dynamics. J. Phys. Chem. Lett. 2019, 10 (12), 3254-3259.

(18) Duboué-Dijon, E.; Mason, P. E.; Fischer, H. E.; Jungwirth, P. Hydration and Ion Pairing in Aqueous Mg2+ and Zn2+ Solutions: Force-Field Description Aided by Neutron 
Scattering Experiments and Ab Initio Molecular Dynamics Simulations. J. Phys. Chem. B 2018, 122 (13), 3296-3306.

(19) Melcr, J.; Martinez-Seara, H.; Nencini, R.; Kolafa, J.; Jungwirth, P.; Ollila, O. H. S. Accurate Binding of Sodium and Calcium to a POPC Bilayer by Effective Inclusion of Electronic Polarization. J. Phys. Chem. B 2018, 122 (16), 4546-4557.

(20) Kennedy, J.; Eberhart, R. 47-Particle Swarm Optimization Proceedings., IEEE International Conference. Proc. ICNN'95 - Int. Conf. Neural Networks 1995, 11 (1), 111117.

(21) Yang, G. A Modified Particle Swarm Optimizer Algorithm. 2007 8th Int. Conf. Electron. Meas. Instruments, ICEMI 2007, 2675-2679.

(22) Poli, R.; Kennedy, J.; Blackwell, T. Particle Swarm Optimization: An Overview. Swarm Intell. 2007, 1 (1), 33-57.

(23) Bonyadi, M. R.; Michalewicz, Z. Particle Swarm Optimization for Single Objective Continuous Space Problems: A Review. Evol. Comput. 2016, 25 (1), 1-54.

(24) Liu, Q. Order-2 Stability Analysis of Particle Swarm Optimization. Evol. Comput. 2014, 23 (2), 187-216.

(25) Zeron, I. M.; Abascal, J. L. F.; Vega, C. A Force Field of Li+, Na+, K+, Mg2+, Ca2+, Cl-, and S O 42 - In Aqueous Solution Based on the TIP4P/2005 Water Model and Scaled Charges for the Ions. J. Chem. Phys. 2019, 151 (13). 